\title{
Active Information and Teleportation *
}

\author{
B J Hiley \\ Theoretical Physics Research Unit, Birkbeck College, University of \\ London, Malet Street, London WC1E 7HX
}

\begin{abstract}
Quantum teleportation is discussed using the notion of active information. We show that this information has an objective meaning and it is this information that is teleported from one particle to another. This information transfer is mediated by the non-local quantum potential. Our approach suggests that there is a new quality of energy operating in the quantum domain and that this energy is non-local. This feature is shared by the gravitational energy in general relativity, a point that has been noted by Penrose who has suggested that this link should be exploited to resolve the measurement problem.
\end{abstract}

\section{Introduction}

In this paper I want to examine quantum teleportation from a point of view that is different from that normally considered [1] [2]. This will enable us to gain a new perspective into what is involved in the process of teleportation. It is clear that, at least in the case where particles are involved, it is not the particle that is transported, but rather the information contained in the wave function. This idea in itself is not new, but the central question that requires clarification is the nature of this information. This paper provides a new insight into this question.

Although the use of 'information' in physics has a long history, there is considerable ambiguity in the precise meaning of the term. When we talk about 'quantum information' this ambiguity becomes greater. What is not clear is whether it is 'information for us' or whether it is some form of objective information that is independent of the observer. I will not attempt

${ }^{*}$ To be Published in The Yearbook of the Institute Vienna Circle, 1999 
to summarise the extended literature on the use of information in physics, as this is unnecessary for the purposes of this paper. (See for example Leff and Rex [3].

To be specific I want to look at quantum teleportation from the Bohm point of view [4]. I will not be concerned with the detail as they have been discussed in Maroney and Hiley [5]. Rather I will discuss the general principles that are involved and show that this approach provides an objective meaning to information. To achieve this I will use the notion of active information introduced by Bohm and Hiley [6],[4]. This notion does not require the presence of an observer to give meaning to the information. What I will show is that it is this active information that is transferred during quantum teleportation.

\section{The Bohm Approach}

Unfortunately there is a great deal of unnecessary emotion generated when 'alternative interpretations' to quantum mechanics are discussed. By now we have so many interpretations, that it must be clear to all that there is some basic ambiguity as to what the formalism is telling us about the nature of quantum processes and their detailed relation to those occurring in the classical domain. The fact that we have very little idea of how to quantise gravity should provide a salutary warning to anyone claiming that we have fully understood the nature of quantum processes. I believe the difficulties the formalism presents is an indication that we need a very deep revision of our physical concepts and that the difficulties will certainly not be removed by a naive return to classical concepts.

My preliminary attempts to explore the quantum formalism with David Bohm centred around the question as to what degree the formalism could support an ontology that would remove some of the paradoxes presented by the standard approach. It has never been my intention to start from a particular metaphysical position and demand that the quantum formalism be forced to support that view at all costs. Rather my aim was to explore the quantum formalism in a different way and to see what consequences followed. This was certainly the motivation of our group at Birkbeck College and our findings have been summarised in Bohm and Hiley [4] and in Holland [7].

The spirit in which I entered into these investigations started with the question "In exactly what way does quantum mechanics differ from classical mechanics?" Any attempts at an answer is clearly hampered by the fact that 
the mathematical structures used in the two theories are so very different. It is true that each formulation carries the same dynamical and geometric symmetries, but they are realised in very different ways. What I found remarkable in Bohms original proposal was that by adopting the Schrödinger representation and using the polar decomposition of the wave function, we obtained a formalism that was very close to the Hamilton-Jacobi formulation of classical mechanics. In retrospect this should not have been too surprising because Schrödinger himself was led to his equation from the HamiltonJacobi formalism in the first place [8].

If we insert the polar decomposition $\psi(\boldsymbol{r}, t)=R(\boldsymbol{r}, t) \exp [i S(\boldsymbol{r}, t) / \hbar]$, into the Schrödinger equation and separate into its real and imaginary parts, we obtain two conservation equations. The first can be regarded as a conservation of energy and the other is a conservation of probability. Thus the complex Schrödinger equation gives rise to two real equations

$$
E=\frac{p^{2}}{2 m}+V+Q
$$

and

$$
\frac{\partial P}{\partial t}+\nabla \cdot\left(P \frac{\nabla S}{m}\right)=0
$$

where $P=R^{2}$

It should be emphasised that these are merely a re-expression of the mathematical content of the Schrödinger equation and that nothing new has been added to the formalism. Therefore clearly no new content can appear. Our purpose was simply to explore all aspects of the formalism and to see if we could gain new insights into the physics that lies behind the formalism. As those who are familiar with the Bohm approach will know, the energy equation (1) is obtained from

$$
\frac{\partial S}{\partial t}+\frac{(\nabla S)^{2}}{2 m}+V(\boldsymbol{r}, t)+Q(\boldsymbol{r}, t)=0
$$

which is the Hamilton-Jacobi equation modified by the presence of an additional term

$$
Q(\boldsymbol{r}, t)=-\frac{\hbar^{2}}{2 m} \frac{\nabla^{2} R(\boldsymbol{r}, t)}{R(\boldsymbol{r}, t)}
$$

To obtain equation (1) from equation (3) it is necessary to use the canonical relations $E=-\frac{\partial S}{\partial t}$ and $\boldsymbol{p}=\nabla S$, which are here to be regarded as subsidiary conditions. 
In the context of equation (1) we can interpret the additional term, $Q(\boldsymbol{r}, t)$ , as a new quality of energy that only appears in the quantum domain and therefore we call it the quantum potential energy. The classical domain is then characterised as the domain in which this additional quality of energy does not appear. In this context it is interesting to note that the gauge fields manifest themselves in this energy and, as we will see later, it is this energy that is involved in the Einstein-Podolsky-Rosen correlations and teleportation.

\section{Trajectories}

Since we have equation (3), which becomes the classical Hamilton-Jacobi equation in the limit when $Q(\boldsymbol{r}, t)$ vanishes, it is only natural to make use of the known properties of the classical equations. In classical mechanics the action, S, enables us to generate a Lagrangian submanifold through the relation $\boldsymbol{p}=\nabla S$ and this equation, in turn, enables us to generate a set of one-parameter curves, which we interpret as an ensemble of possible particle trajectories. When we go over to the case where $Q(\boldsymbol{r}, t)$ is not zero, $\boldsymbol{p}=\nabla S$ will again generate a set of one-parameter curves, and furthermore these curves will clearly coincide with the classical curves as $Q(\boldsymbol{r}, t)$ approaches zero. This relation suggests that we should regard these curves generalised trajectories'.

At this stage there are no metaphysical assumptions as to what these trajectories may mean physically. My own inclination has been to regard these curves as the centre of some active process, which, for convenience, we regard as the path of a 'quantum particle'. However for simplicity we can retain the notion of a particle with a well-defined position and momentum and regard these curves as the paths along which such a particle would actually move. The trajectories then show how the particles must behave if we are to obtain results that are consistent with the standard approach. The difference between these trajectories and there classical counterparts can then be attributed to the presence of the new quality of energy contained in the quantum potential.

The fact that there is no experiment that can be made to distinguish between standard quantum mechanics and any approach exploiting these one-parameter solutions of equation (3) has led to the argument that the trajectories merely add 'metaphysical baggage' that we do not need. There is a great deal of attraction for this point of view because thinking in terms 
of trajectories brings in all our classical prejudices that could possibly lead to confusion. However it is very difficult not to think of quantum phenomena in terms of 'particles following trajectories' so it is better to have a view that is consistent with the quantum formalism rather than to 'guess' what these trajectories could be. As I have said above and will repeat again, quantum phenomena require us to think in a radical new way, a way in which we will have to ultimately give up both the notion of particles and fields. Nevertheless, as we explained in our book, Bohm and Hiley [4], by adopting the simplifying assumption of a particle with a well-defined position and momentum, we can obtain a consistent interpretation of the quantum formalism that does not contain many of the perplexing paradoxes that one faces daily in the standard approach.

Of course one could argue that these trajectories are metaphysical simply because it is not possible to measure position and momentum simultaneously. Therefore there is no way to directly verify the presence of these trajectories so why introduce them in the first place? Indeed this objection was first raised by Pauli [9]. One could counter this by arguing that the wave function is not directly observed either and therefore it too should be regarded as 'metaphysical'. However such an objection is never raised because the wave function is used to calculate probabilities that are consistent with the experiment results and therefore vital for the theory.

A similar argument could be applied to the trajectories. They show how you could retain the notion of a localised particle and explain how the resulting probability distributions arise without violating the uncertainty principle. Indeed both of these arguments are consistent with Einstein's proposals that it is of no importance if you cannot verify directly certain elements in a theory as long as you can produce verifiable consequences that depends on their existence. There are many other notable examples of such quantities in physics; for example space-time points, quarks and so on.

Englert et al [10] have tried to demonstrate that in certain situations, these one-parameter curves will be different from the 'quantum trajectories' inferred from the results of a particular interferometer. Unfortunately although their claims are supposed to originate from orthodox quantum mechanics they draw conclusions about which path a particle will take in an interferometer. But the notion of a 'particle path' in an interferometer is just what is denied in orthodox quantum mechanics! Our approach has mathematically well-defined one-parameter curves which we interpret as particle trajectories'. These one-parameter curves exist regardless of what interpretation we give them. The fact that these curves have properties that are 
very different from those that we would expect from a classical physics, is not a sound reason for rejecting them; we would not expect 'quantum particles' to behave like classical particles. An explanation of why these particles behave the way they do was given by Dewdney et al [11] and depends crucially on the novel properties of the quantum potential. If the properties of this potential are ignored, the approach using $p=\nabla S$ will seem ad hoc, but that is no reason for rejecting the approach. (A detailed consideration of these issues will be presented in Hiley [12]

Perhaps the strongest argument suggesting that these trajectories are not merely metaphysical comes from a specific example in which the quantum potential is a function of time. This example emerged from a consideration of the inflationary universe [13]. A simplified model of inflation consists of an 'upside-down' harmonic oscillator potential with a particle (the 'Universe) initially in a Gaussian wave-packet sitting on top of the classical potential. Inflation corresponds to the particle rolling down the potential. As it rolls down, the quantum potential becomes smaller and smaller and, in the long-time limit, it becomes negligible. What we showed was that any initial quantum trajectory evolves smoothly into a corresponding classical trajectory as the particle rolls down the potential. If we insist that the quantum trajectory is metaphysical, how is it possible for a 'metaphysical trajectory to become 'real' as a process evolves in time?

I want to make it absolutely clear here that I am not concluding from these arguments that the quantum particle actually is a 'small lump of substance' changing mechanically as the process develops in time. Something much subtler is involved. Nevertheless as I have remarked above, we can think of this process as being represented as a particle to within a certain approximation without arriving at a contradiction. When we go deeper and use field theory, a more complex process is revealed and clearly the simple particle picture must be modified in some radically new way.

I want also to stress that I do not believe that, even at the particle level we can regard the process as mechanical. As I will show in the next section, when the quantum potential is examined more closely it does not have the properties of a classical potential and suggests that something subtler is involved. This view has been explained fully in our book "The Undivided Universe" [4].

It should be noted that the views expressed in our book differ very substantially from those of Dürr et al [14] who have developed an alternative theory. It was very unfortunately that they chose the term Bohmian mechanics' to describe their work. When Bohm first saw the term he remarked, 
"Why do they call it 'Bohmian mechanics'? Have they not understood a thing that I have written?" He was referring not only to our article [6], but also to a footnote in his book Quantum Theory in which he writes, "This means that the term 'quantum mechanics' is a misnomer. It should, perhaps, be called "quantum nonmechanics"' [15]. It would have been far better if Dürr et al [14] had chosen the term 'Bell mechanics'. That would have reflected the actual situation far more accurately.

\section{Information Dynamics}

I now want to take a closer look at how we are to understand the structure underlying the modified Hamilton-Jacobi equation (3). As I have remarked above this equation cannot be interpreted consistently as an equation describing a particle evolving mechanically [6], [4], [16]. The quantum potential is not like a classical potential. It has properties that suggest a more 'organistic process is involved. Such a proposal is not new, and has already been suggested by Whitehead [17], but such ideas have not been accepted in physics. Most physicists prefer to be "metaphysically neutral" about such questions although, in fact, they rely implicitly on a particle model. However I feel that the next stage of the development of our ideas will necessarily require metaphysical assumptions to motivate new approaches from which new mathematical structures can emerge. These structures will have new experimental predictions which will provide the necessary tests. The success or failure of this mathematics will then enable physicists once again to become "metaphysically neutral".

Let me begin by summarising the main points that lead to the conclusion that we must adopt a non-mechanical view. Firstly the quantum potential has no external source. Since it is derived mathematically from the real part of the wave function, we must regard it as being generated with the particle in what is conventionally called the 'preparation device'. In a sense we can regard the particle and the quantum field as distinguished aspects of a single process. This argument becomes more plausible if we assume that fundamentally particles are excited states of a basic underlying field. Our proposal is that the source of the quantum potential is part of this underlying field. If this proposal is correct then what would be involved here is some kind of self-organising process.

Secondly the quantum potential is not changed by multiplying the field, $\psi$, by a constant. This can be seen by examining the mathematical form of 
the quantum potential given by equation (4). This means that the quantum potential is independent of the magnitude of $\psi$ and so is independent of the field intensity. This in turn means that its effect can be very large even when the amplitude of the field is very small. Because of this, the effect of the potential need not fall off as the distance increases and this is just the property required for an explanation of the EPR correlations.

Thirdly the quantum potential carries information about the environment in which the particle finds itself. For example, in the electron two-slit experiment, the quantum potential carries information about the two slits, their size, shape and distance apart. Thus it carries information about the whole experimental arrangement.

This conclusion should come as no a surprise because it has been stressed by Bohr on more than one occasion. For example he writes "I advocate the application of the word phenomenon exclusively to refer to the observations obtained under specific circumstances, including an account of the whole experimental arrangement." [18]. Thus rather than our interpretation contradicting Bohrs position, it actually confirms some of his deeper insights. I would go further and add that our approach actually gives a clearer meaning to this statement. Thus our approach is not in total opposition to the standard approach, but actually clarifies aspects of the theory by providing a different perspective from which to explore the quantum processes.

These properties show how the quantum potential is essentially different from a classical particle, so different that we must conclude that it does not give rise to a mechanical force in the Newtonian sense. Thus while the Newtonian potential drives the particle along the trajectory, the quantum potential organises the form of the trajectories in response to the experimental conditions. In other words information about the boundary conditions is playing a vital role in the overall dynamics. Again this should not surprising since in solving the Schrödinger equation we must include the boundary conditions and boundary conditions are essential to determine the correct solutions. Our approach simply translates the information contained in the boundary conditions into information determining the kinematics of the evolving process.

\section{Active Information}

The word 'information' is used in various ways in physics. The most common usage is in terms of Shannon information. This is rather a technical use of 


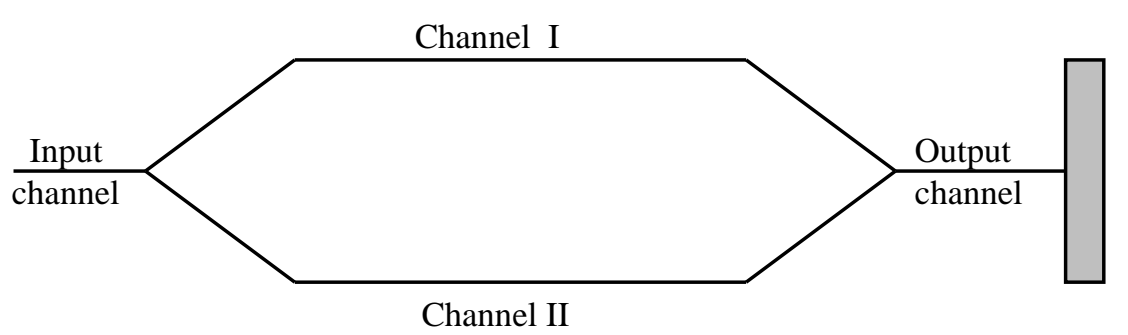

Figure 1: Schematic interference device

the word which is actually better understood in terms of the 'capacity for carrying information' rather than information per se. Thus in designing a communications systems, we need to know how many bits can be carried per second by a particular processor. The potential meaning of the messages to be carried is totally irrelevant as far as these design factors are concerned. The question of meaning is not addressed because that is generally perceived as being something specific to human activity and therefore outside the considerations of physics.

However although the common usage of the word information does carry a strong human element, an examination of the etymological origins of the word show that it has a more general meaning. For example, the central stem (forma) carries the primary meaning of a visible form, an outward appearance, or a shape. So informo (or informare) signifies the action of forming, fashioning or bringing a certain shape or order into something. Thus the root of the word has to do with the activity of shaping or putting form into a given process, be it a physical process or one that involves human activity. We use the word 'information' in this sense. Thus for us the word 'information' describes the objective process that results from a dynamics constrained in an irreducible way by the environment. In this way we can discuss an objective process free of all human connotations.

In applying the notion of information to physical processes, we have found it necessary to distinguish between three categories of information, active information, passive information and inactive information. To illustrate the need for these distinctions consider the general two-beam interference experiment shown in figure 1. 


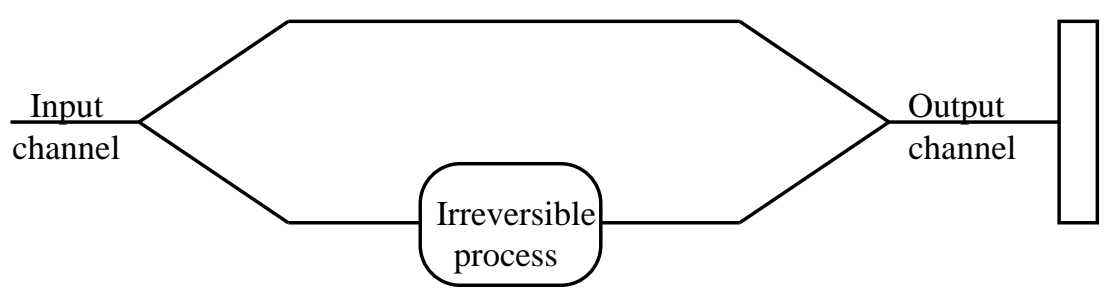

Figure 2: Schematic interference device with detector

If the particle travels in channel I, only the information in channel I is 'active' (literally 'acts on the particle'). There is a quantum potential present in channel II but this does not act on the particle. However it cannot be discarded because we need it to be present later in the final output channel. Thus we regarded this information in this channel as passive'. If the particle goes through channel II, the information in channel I becomes passive, while the information in channel II becomes active. In either case, when the particle enters the output channel, the information from both channels is combined and the particle trajectories modified to produce the required interference pattern.

Does information ever become inactive? One place where it can become inactive is in an irreversible process. To see how this works consider figure 2 above:

The wave function arriving in the output channel here would be

$$
\Psi_{\text {output }}=a \psi_{1}(\boldsymbol{r}, t) \chi_{\text {unfired }}+b \psi_{2}(\boldsymbol{r}, t) \chi_{\text {fired }}
$$

By 'fired' we simply mean that the detector has registered the passing of the particle. This registration requires a macroscopic movement of some of the particles in the device induced by the process being triggered by the passing particle. Even if the state of the passing particle has only been changed slightly, there will be no interference effects in the output channel because the information in the 'unfired channel' is no longer active. It has become 'inactive' or even 'deactivated'.

To see in detail how this works, we must evaluate the quantum potential 
in the output channel after the device has fired. To do this we must calculate the quantum potential from wave function (5) using the final positions of all the particles involved in the process. Since in the final state, the detector particles have moved over macroscopic distances so their wave packets no longer overlap, the contribution from the 'unfired' state is zero when evaluated over the detector particles when they are in their 'fired' position. If the device has not fired, then the contribution from the 'fired' state would be zero.

In order to obtain a contribution from the 'unfired' state in the first case, it would be necessary to return all the particles in the 'fired' state to their 'unfired' positions in some way. But this is impossible in practice since it is not possible to make all of the particles in such a cascade return to their 'unfired positions. Thus it is the presence of an irreversible process that renders the information 'inactive'.

This leaves the case when the detector did not fire. Here when one evaluates the quantum potential using the positions of the 'unfired' state, we find that the contribution from the second term in equation (5) gives a zero contribution. Thus no interference will be seen in the output channel. So it does not matter whether the particle passes along channel I or channel II, no interference will be seen in the output channel even if the detector does not fire.

In this approach then, there is no 'collapse' of the wave function. The information associated with the wave function simply becomes inactive and cannot be reactivated. All of this occurs specifically without the intervention of the observer. Thus by using this objective concept of information we have avoided the measurement problem.

\section{Non locality}

It is by now well known that if we extend our ideas to consider many-particle systems, the quantum potential will exhibit non-local features for groups of particles described by an entangled wave function. It was this aspect of the approach that drew Bell's attention to the general question of nonlocality in quantum mechanics [19]. He asked whether all approaches that attribute local properties to individual particles and reproduce the results of quantum mechanics must exhibit non-local properties. The result of Bell's work clearly shows non-locality is not specific to the Bohm approach, but is in fact quite general [20]. 
We can see how this non-locality arises in the Bohm approach by considering the expression for the quantum potential. For example, for a twoparticle system described by an entangled wave function $\Psi\left(\boldsymbol{r}_{1}, \boldsymbol{r}_{2}, t\right)$, the quantum potential is

$$
Q\left(\boldsymbol{r}_{1}, \boldsymbol{r}_{2}, t\right)=-\frac{\hbar^{2}}{2 m} \frac{\left(\nabla_{1}^{2}+\nabla_{2}^{2}\right) R\left(\boldsymbol{r}_{1}, \boldsymbol{r}_{2}, t\right)}{R\left(\boldsymbol{r}_{1}, \boldsymbol{r}_{2}, t\right)}
$$

which clearly exhibits non-local features. Although this non-local connection exists and may evolve in time, there is no hint of anything 'flowing' between the two particles. The energy in the quantum potential is not localised and is determined by the overall experimental conditions. Since these are global features, it would be surprising to find all the energy involved in these correlations to be localised. In other words we should expect these global features to be reflected in a non-localised energy.

Of course if we approach this idea from the standpoint of classical physics, we would strongly object to any form of non-local energy. However before objecting too strongly, we should recall the nature of gravitational energy. In general relativity energy is needed to curve spacetime but this energy cannot be localised. The argument is very simple (See Misner et al [21]) and is as follows: - Einstein's equivalence principle plays a pivotal role in general relativity. It says that one can always find a frame in which all local gravitational fields disappear. If there are no local gravitational fields, there can be no local gravitational energy, but this does not mean that there is no gravitational energy, it just means that it cannot be localised.

The similarity between the non-local gravitational energy and the nonlocal correlation energy involved in the Einstein-Podolsky-Rosen effect has already been noted by Penrose [22]. He has tried to make use of this common feature to provide an alternative way of solving the measurement problem. Whether his proposals do solve this problem are necessary or whether our proposals out lined above are sufficient is something that can only be answered by experiment. To this end Penrose has proposed a specific experiment to test his ideas. Whatever the outcome of this experiment there nevertheless seems to be a deep connection between the non-local gravitational energy and the non-local EPR correlation energy.

Before leaving this subject I would like to reaffirm the fact that the Bohm approach offers an explanation of the EPR correlation. It does so by explicitly using the non-local properties of the quantum potential. I would like to emphasise once again that this feature is not what we would expect of a classical mechanical explanation. In fact our approach has more in common 
with Bohr's answer to the original EPR objections. He wrote "From our point of view we now see that the wording of the above mentioned criterion of physical reality proposed by Einstein, Podolsky and Rosen contains an ambiguity as regards the meaning of the expression "without in any way disturbing a system." Of course there is no question of a mechanical disturbance of the system under investigation during the last critical stage of the measuring procedure. But even at this stage there is essentially the question of an influence on the very conditions which define the possible types of predictions regarding the future behaviour of the system." [23] [Bohr's italics, but my emphasis on the words "mechanical and "influence.]

Note that in our view also there is no mechanical force because the quantum potential does not operate mechanically. The meaning of the word 'influence' used by Bohr is unclear, but it can be understood in terms of active information, which, as we have explained, contains information about the overall experimental conditions and it is these conditions that are responsible for the correlations. Furthermore since we are dealing with a global effect there is no direct energy transfer involved.

\section{Quantum Fusion and Fission}

In this final section I would like draw attention to some questions that I began raising in the 1970s because these lie at the heart of quantum teleportation effects. Unfortunately I could not get people to take these ideas seriously at that time and I only wrote them up in a very obscure conference proceedings ([24]). A modified version of these ideas were later included in Bohm and Hiley [4]. It seemed to me that the following basic question needed to be answered. "What physical processes allow a set of single particle quantum potentials to become 'fused' into a group organised by an inseparable quantum potential?" In other words how could we produce the fusion

$$
Q_{1}\left(\boldsymbol{r}_{1}, t\right) Q_{2}\left(\boldsymbol{r}_{2}, t\right) \ldots Q_{n}\left(\boldsymbol{r}_{n}, t\right) \rightarrow Q\left(\boldsymbol{r}_{1}, \boldsymbol{r}_{2}, \ldots . \boldsymbol{r}_{n}, t\right)
$$

Alternatively we could consider the opposite process, namely, fission where

$$
Q\left(\boldsymbol{r}_{1}, \boldsymbol{r}_{2}, \ldots . \boldsymbol{r}_{n}, t\right) \rightarrow Q_{1}\left(\boldsymbol{r}_{1}, t\right) Q_{2}\left(\boldsymbol{r}_{2}, t\right) \ldots Q_{n}\left(\boldsymbol{r}_{n}, t\right)
$$

One thing is clear; fission always occurs in a measurement process. Fusion, on the other hand, is rarer. Of course we do have some examples as 
in the pair production of photons in the atomic cascade processes used by Aspect et al [25] in their experiments to test the Bell inequalities. Other possibilities include the pairs of g-rays produced in positronium decay, or photons produced in parametric downconversion.

However there are more interesting processes. For example, quantum teleportation would require the process

$$
Q_{1}\left(\boldsymbol{r}_{1}, t\right) Q_{2}\left(\boldsymbol{r}_{2}, \boldsymbol{r}_{3}, t\right) \leftrightarrow Q_{1}\left(\boldsymbol{r}_{3}, t\right) Q_{2}\left(\boldsymbol{r}_{1}, \boldsymbol{r}_{2}, t\right)
$$

To show how this works, we calculate $Q_{1}\left(\boldsymbol{r}_{1}, t\right) Q_{2}\left(\boldsymbol{r}_{2}, \boldsymbol{r}_{3}, t\right)$ from the wave functions (written as kets for convenience)

$$
|\Psi\rangle_{123}=\left(a|1\rangle_{1}+b|0\rangle_{1}\right)\left(|1\rangle_{2}|1\rangle_{3}+|0\rangle_{2}|0\rangle_{3}\right)
$$

Thus our quantum potential can be written in the form

$$
Q\left(\boldsymbol{r}_{1}, \boldsymbol{r}_{2}, \boldsymbol{r}_{3}\right)=Q_{1}\left(\boldsymbol{r}_{1}, a, b\right) Q_{23}\left(\boldsymbol{r}_{2}, \boldsymbol{r}_{3}\right)
$$

Here the coefficients a and $\mathrm{b}$ are associated with the quantum potential acting on particle 1 . This means that initially the information characterised by the pair $[\mathrm{a}, \mathrm{b}]$ actively operates on particle 1 alone. At this stage the behaviour particle 3 is independent of $[\mathrm{a}, \mathrm{b}]$ as would be expected. To proceed we simply re-write equation (10) in the form

$$
\begin{aligned}
2|\Psi\rangle_{123} & =\left|\Phi^{+}\right\rangle_{12}\left(a|0\rangle_{3}+b|1\rangle_{3}\right)+\left|\Phi^{-}\right\rangle_{12}\left(-a|0\rangle_{3}+b|1\rangle_{3}\right) \\
& +\left|\Psi^{+}\right\rangle_{12}\left(b|0\rangle_{3}+a|1\rangle_{3}\right)+\left|\Psi^{-}\right\rangle_{12}\left(-b|0\rangle_{3}+a|1\rangle_{3}\right)
\end{aligned}
$$

where we have defined the Bell states as

$$
\begin{aligned}
& \sqrt{2}\left|\Psi^{ \pm}\right\rangle_{12}=\left[|1\rangle_{1}|0\rangle_{2} \pm|0\rangle_{1}|1\rangle_{2}\right] \\
& \sqrt{2}\left|\Phi^{ \pm}\right\rangle_{12}=\left[|1\rangle_{1}|1\rangle_{2} \pm|0\rangle_{1}|0\rangle_{2}\right]
\end{aligned}
$$

The final step requires actually carrying out a Bell measurement. This involves coupling particle 1 to particle 2 by some suitable process. The coupling interaction will involve an appropriate interaction Hamiltonian, which will depend on the particular apparatus used. During this interaction a new quantum potential will be generate that will couple all three particles to the measuring apparatus. This is the fusion aspect of the process, which occurs no matter how far particle 3 is from particle 2 . When the interaction 
is over the final wave function will undergo fission and end up in one of the four terms appearing in equation (12). This will produce a final quantum potential

$$
Q\left(\boldsymbol{r}_{1}, \boldsymbol{r}_{2}, \boldsymbol{r}_{3}, \boldsymbol{r}_{y}\right)=Q_{12}\left(\boldsymbol{r}_{1}, \boldsymbol{r}_{2}, \boldsymbol{r}_{y}\right) Q_{3}\left(\boldsymbol{r}_{3}, \boldsymbol{r}_{y}, a, b\right)
$$

Here $\boldsymbol{r}_{y}$ is the relevant set of variables for the measuring instrument. Thus after the measurement the information contained in a and b has now been encoded in $Q_{3}\left(\boldsymbol{r}_{3}, \boldsymbol{r}_{y} \cdot a, b\right)$ which provides the active information for particle 3 . In other words the information that was active in particle 1 has been transferred to particle 3 so that the behaviour of particle 3 will be different after the measurement. Thus it is the active information that has been transferred between the two particles. More details of this analysis can be found in Maroney and Hiley [5]

\section{Conclusion}

In this paper we have examined the implications of the real part of the Schrödinger equation when it is written in the form shown in equation (3). This equation can be regarded as an expression for the conservation of energy if we identify the extra term, Q, as a new form of energy that arises only in the quantum domain. As we have shown in detail elsewhere [4], this new quality of potential energy enables us to account for the essential differences between classical and quantum behaviour in ways that are independent of the observer. Furthermore it provides a coherent account of quantum effects, freeing us from many of the paradoxes that arise in the usual interpretation.

In order to provide a consistent account, we have to give the one-parameter solutions of equation (3) special significance as the centre of some form of 'activity', which to a first approximation, we can regard as the 'locus of a quantum particle'. We then find that this new energy, just like the energy of the gravitational field, is non-local. Contrary to the claims of Dürr et al [14], we are unable to give our approach a consistent mechanical interpretation. Rather we find it necessary to introduce the new notions of active and passive information [6] and [4].

These notions of information, together with the non-locality, enable us to explain the EPR correlations in a manner that is not dissimilar from Bohr's original explanation. Furthermore if we apply these notions to quantum teleportation, we find that it is the active information that is transferred 
from one particle to another and that this transfer is mediated by the nonlocal quantum potential.

Let me stress again that this active information is 'information for the particle' and at this stage has nothing to do with 'information for us'. Since our discussion above only involves a single pure state, there is no question of this information being identified with Shannon information. Shannon information will appear only when we consider a source that could be prepared in one of a number of orthogonal wave functions, each of which could be transferred separately. Here we have sufficient complexity to enable us to discuss channel capacities in terms of qubits in the usual way, but what is actually transferred is objective active information.

\section{Acknowledgements}

I would like to thank O. Cohen and O. Maroney for helpful discussions in clarifying these ideas.

\section{References}

[1] C. H. Bennett, G. Brassard, C. Crpeau, R. Jozsa, A. Peres and W. K. Wooters, Phys. Rev. Lett. 70, 1895-99,1993.

[2] D. Bouwmeester, J-W Pan, M. Daniell, H. Weinfurter and A. Zeilinger, Phys. Rev. Lett. 82, 1345-1349, 1999.

[3] H. S. Leff and A. F. Rex, Maxwell's demon : entropy, information, computing. Hilger, Bristol, England, 1990.

[4] D. Bohm and B. J. Hiley, The Undivided Universe: an Ontological Interpretation of Quantum Theory, Routledge, London, 1993.

[5] O.Maroney and B. J. Hiley, Quantum State Teleportation understood through the Bohm Interpretation, To appear in Foundations of Physics, 1999 .

[6] D. Bohm and B. J. Hiley, Phys. Reports, 144, 323-348, 1987.

[7] P. Holland, The Quantum Theory of Motion, Cambridge University Press, Cambridge, 1993. 
[8] E. Schrödinger, Ann. der Physik, 79, 361,1926. English Translation in Collective Papers on Wave Mechanics, Blackie and Son, London, 1928.

[9] W. Pauli, Louis de Broglie, Physicien et Penseur, Ed, A. George, p. 33, Editions Albin Michel, Paris, 1953.

[10] B. Englert, M. O. Scully, G. Sssman and H. Walther, Z. Naturforsch. 47a, 1175-1186, 1992.

[11] C. Dewdney, L. Hardy and E. J. Squires, Phys. Lett. 184, 6-11, 1993.

[12] B. J. Hiley, R. Callaghan and O. Maroney, Quantum trajectories, Real, Surreal or an approximation to a Deeper Process? (To be published, 1999)

[13] B. J. Hiley and A. H. Aziz Muft, in Fundamental Problems in Quantum Physics, ed. M. Ferrero and A. van der Merwe, 141-156, Kluwer, Dordrecht, 1995.

[14] D. Drr, S. Goldstein and N. Zanghi, J. Stat. Phys. 67, 843-907, 1992.

[15] D. Bohm,QuantumTheory, p. 167, Prentice-Hall, Englewood Cliffs, N.J.1951.

[16] B. J. Hiley, in The Philosophy of Vacuum, ed. by S. Saunders and H. Brown, 217-249, Cambridge Uni. Press, Cambridge, 1991.

[17] A. N. Whitehead, Process and Reality, Harper and Row, New York, 1957.

[18] N. Bohr, Atomic Physics and Human Knowledge, p. 64 Science Editions, New York,1961.

[19] J. S. Bell, Rev. Mod. Phys. 38, 447-52,1966.

[20] J. S. Bell, Physics, 1, 195-200,1964.

[21] C. W. Misner, K. S. Thorne and J. A. Wheeler, Gravitation, pp. 466468, Freeman, San Francisco,1970.

[22] R. Penrose, in The Nature of Space and Time, ed, S. Hawking and R. Penrose, 61-73, Princeton University Press, New Jersey, 1996.

[23] N. Bohr,Phys. Rev., 48, 696, 1935. 
[24] B. J. Hiley, Quantum Mechanics and Reality, in De Aard van de Wekelijkheid, ed. J.L. Grashuis,J.A.F. Ooomen, R. Stoeckart and G.S. van de Ven, pp.36-37, Erasmus University, Rotterdam, 1983.

[25] A. Aspect, J. Dalibard and G. Roger,Phys. Rev. Letts., 49, 1804, 1982. 